\title{
Elementary Scattering Theory
}

\section{For X-ray and neutron users}

D.S. SIVIA

St John's College, Oxford 


\section{Contents}

I Some preliminaries 1

1 Studying matter at the atomic and molecular level 3

1.1 Length scales and logarithmic axes 3

1.2 Resolution, magnification and microscopy 6

1.3 Structure, dynamics and spectroscopy 9

1.4 Atomic building blocks and interactions 11

1.4.1 The basic structure of the atom 12

1.4.2 The fundamental forces of nature 13

1.4.3 Probing matter by scattering particles 14

1.5 Energy, length and temperature scales 16

$\begin{array}{ll}1.6 & \text { A table of useful constants } \\ & 18\end{array}$

2 Waves, complex numbers and Fourier transforms 19

2.1 Sinusoidal waves 19

2.1.1 The direction of propagation 21

2.1.2 The principle of superposition 23

2.2 Complex numbers 25

2.2 .1 Definition 25

2.2.2 Basic algebra $\quad 26$

2.2.3 The Argand diagram 27

2.2.4 The imaginary exponential 27

2.3 Fourier series 29

2.3.1 Orthogonality and the Fourier coefficients 30

2.3.2 The complex Fourier series 32

2.4 Fourier transforms 32

2.4.1 Convolution theorem 34

2.4.2 Auto-correlation function 36

2.5 Fourier optics and physical insight 38

2.5.1 Young's double slit 40

2.5.2 A single wide slit 41

2.5.3 A diffraction grating $\quad 42$

2.5.4 The convolution theorem in action 43

2.5 .5 Multi-dimensional generalization 44

2.6 Fourier data analysis 48

2.6.1 The phase problem 48

2.6.2 Truncation effects and windowing 50

2.6.3 Noise and probability theory 52 
3 The basics of X-ray and neutron scattering 63

3.1 An idealized scattering experiment 63

3.1.1 Elastic scattering and momentum transfer $\quad 64$

3.1.2 The differential cross-section 66

3.1.3 Elastic versus total scattering $\quad 67$

3.2 Scattering by a single fixed atom $\quad 67$

3.2.1 Nuclear scattering lengths $\quad 69$

3.2.2 Atomic form factors $\quad 71$

3.2.3 Magnetic form factors $\quad 73$

3.2.4 Scattering cross-sections 75

3.3 Scattering from an assembly of atoms 77

3.3.1 Scattering density and Fourier transforms $\quad 79$

3.3.2 Temperature and Debye-Waller factors 81

3.3.3 Coherent and incoherent scattering $\quad 82$

3.3.4 Mixed scattering events 84

3.4 X-rays and synchrotron sources $\quad 84$

3.4.1 Wigglers and undulators $\quad 88$

3.5 Reactors and pulsed neutron sources $\quad 89$

3.5.1 The time-of-flight technique 91

4 Surfaces, interfaces and reflectivity 93

4.1 Reflectivity and Fourier transforms 93

4.1.1 Substrate only 98

4.1.2 One uniform layer $\quad 100$

4.1.3 A short multilayer 101

4.1.4 A phaseless Fourier ambiguity 102

4.2 Reflectivity and geometrical optics 104

4.2.1 Refractive index 105

4.2.2 A single boundary 105

4.2.3 Multiple boundaries 108

4.3 X-rays, neutrons and other techniques 110

5 Small-angle scattering and the big picture 113

5.1 Diffraction and length scales 113

5.2 Size, shape and molecular form factors 114

5.2.1 Absolute intensities 118

5.2.2 The Guinier approximation 119

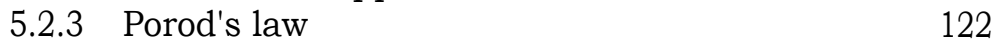

5.2.4 Polydispersity 124

5.3 Assemblies and correlations $\quad 125$

$\begin{array}{ll}\text { 5.3.1 Orientational alignment } & 125\end{array}$

5.3.2 Positional correlation $\quad 126$

$\begin{array}{lll}5.4 & \text { Pair-distribution function } & 127\end{array}$ 
6 Liquids and amorphous materials 131

6.1 The middle phase of matter 131

6.2 Radial distribution functions 132

6.3 Structure factors 134

6.3.1 Partial structure factors 137

6.4 Comparison with small-angle scattering 139

6.5 The Placzek correction 139

7 Periodicity, symmetry and crystallography 141

$\begin{array}{lll}7.1 & \text { Repetitive structures and Bragg peaks } & 141\end{array}$

7.1.1 Atomic planes and Bragg's law 144

7.1.2 Simple consequences and applications $\quad 146$

$\begin{array}{lll}7.2 & \text { Patterns and symmetries } & 147\end{array}$

7.2.1 Reality and Friedel pairs 147

$\begin{array}{lll}7.2 .2 & \text { Centrosymmetry and reality } & 148\end{array}$

7.2.3 Space groups and systematic absences 148

7.2.4 Geometry and space groups 150

7.2.5 Symmetry and statistics 151

7.3 Circumventing the phase problem 151

7.3.1 Patterson maps 152

7.3.2 Heavy atoms and partial structure 153

7.3.3 Isomorphous replacement 154

7.3.4 Direct methods and prior knowledge $\quad 155$

$\begin{array}{lll}7.4 & \text { Powdered samples } & 156\end{array}$

$\begin{array}{lll}7.4 .1 & \text { Texture } & 158\end{array}$

$\begin{array}{lll}7.4 .2 & \text { Twinning } & 159\end{array}$

7.4.3 Fibre diffraction $\quad 160$

$\begin{array}{lll}7.5 & \text { Magnetic structures } & 160\end{array}$

III Inelastic scattering 163

8 Energy exchange and dynamical information 165

8.1 Experimental considerations 165

8.1.1 The partial differential cross-section $\quad 166$

8.1.2 Triple-axis spectrometer 167

8.1.3 Time-of-flight instruments 168

8.2 Scattering from time-varying structures 169

8.2.1 Elastic scattering $\quad 170$

8.2.2 Space-time correlation function $\quad 170$

8.2.3 Total scattering 171

8.2.4 Coherent and incoherent scattering $\quad 172$

8.3 A quantum transitions approach 173

9 Examples of inelastic scattering $\quad \mathbf{1 7 5}$

9.1 Compton scattering $\quad 175$

9.1.1 The impulse approximation 177 
xii Contents

9.1.2 Single particle wave function 178

$\begin{array}{lll}9.2 & \text { Lattice vibrations } & 179\end{array}$

9.2.1 Heat capacities $\quad 182$

9.2.2 Spin waves 183

$\begin{array}{lll}9.3 \text { Molecular spectroscopy } & 184\end{array}$

9.3.1 Quasi-elastic scattering 185

9.3.2 Energy resolution and time-scales 186

$\begin{array}{lr}\text { A Discrete Fourier transforms } & 187\end{array}$

B Resonant scattering and absorption $\quad 191$

$\begin{array}{lr}\text { References } & 193\end{array}$

$\begin{array}{lr}\text { Index } & 197\end{array}$ 